\title{
Interactive comment on "Refining the sea surface identification approach for determining freeboards in the ICESat-2 sea ice products" by Ron Kwok et al.
}

\section{Anonymous Referee \#1}

Received and published: 8 August 2020

This article documents the improvements of sea-surface identification with ICESat-2 altimetric measurements for sea ice, and the ensuing changes in the new release (i.e., ver. 3) of sea ice freeboard. Specifically, photon rate on dark leads can be reduced by cloud, which may cause mis-categorization as sea level tie points and result in underestimation of sea ice freeboard. A case study with collocating airborne measurements is carried out, and a correction is introduced to utilize only specular returns for lead detection and sea-level computation. Basin-scale effects on freeboard estimation is also studied for Arctic and Antarctic retrieval. Given the importance of the ICESat-2 measurements for the ongoing and future observation of sea ice thickness, this work is an important and timely update for the polar research community. The article is also 
well-written, and the presentation of the results are very good. However, I find several aspects that can be improved especially in the results and analysis part. My major comments and questions are as follows.

Major comment 1: one key analysis that I suggest to add, is the study/validation with collocating tracks of ICESat-2 and OIB. For example, the 4 OIB tracks as used in Kwok et al (2019), which showed very good consistency between OIB and IS-2 elevation, can be utilized. In fact, in Kwok et al. (2019), the effect on freeboard retrieval is discussed. But key info (including the systematic differences of freeboard, as shown Tab. 1 of the reference) may be different from the current version of the IS-2 data, including protocols, etc. Besides the basin-scale comparisons as in Sec. 5 of the manuscript, the study on the fine spatial scale may be more informative of the performance of the new treatments, as well as the consistency with OIB which feature a sea level retrieval with more comprehensive measurements. This revision is also necessary to improve the overall content of Sec. 5, which in its current form is too short and contains limited result and analysis.

Major comment 2: previous release (ver. 2) of sea ice freeboard (ATL07) contains swath-wide freeboard estimations, by using sea-level estimations across all 3 strong beams. This effectively mitigates the problem of missing parts of the beam ground segments when ssh is computed within a single beam. Besides, there also exists effect on the freeboard value for each beam segment. Since about $10 \%$ to $20 \%$ coverage is lost due to the new treatments (that excludes dark leads), this cross-beam processing could be potentially important in salvaging more data. The authors are suggested to clarify both ends of the comparison. First, whether the previous version of freeboard (R002) is based on per-beam freeboard OR swath-wide freeboard. Second, whether the new version of freeboard (R003) is based on per-beam freeboard OR swath-wide freeboard. Furthermore, whether there is a swath-wide freeboard estimation in the newly-released version of the ATL data.

Major comment 3: the discussion of the potential effect of the new scheme on sea ice \\ Discussion paper}

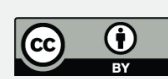


thickness is suggested to be added. During wintertime (first row in Fig. 5 and third row in Fig. 6), the average total freeboard increased by about $3 \mathrm{~cm}$ with the new treatments. This directly translates into about $30 \mathrm{~cm}$ systematic increase in sea ice thickness (if the snow conditions stay the same). Does this help to mitigate the observed differences between ICESat-2 thickness and CryoSat-2 estimations? [Fig. 14 of Petty et al (2020), and related text contents] The lack of detectable leads can be especially prominent within the packed ice region in the Arctic. What is the impact of losing $10 \%$ to $20 \%$ ICESat-2 measurements on the estimation of ice thickness in this region and the total volume?

\section{Minor comments:}

The analysis has been focused on strong beams of IS-2. What is the effect on the retrieval of weak beams? Does the loss of $10 \%$ to $20 \%$ of coverage include statistics from weak beams? Given the lower power of these beams (\#2, \#4 and \#6) and the lower photon rates, is the data loss rate higher in weak beams than strong beams with the new treatments in R003?

Line 33, page 4: delete extra '. after 'use'.

Line 11, page 5: "the second percentile in ...".

Line 12, page 5: “... for smooth surfaces in IS-2 the retrieved heights" should be "... for smooth surfaces in the retrieved heights of IS-2".

Line 25, page 5: the definition of $\mathrm{H} \_$lower is missing.

Line 11, page 6: add the missing “.”.

Line 17, page 8: delete the extra space.

Printer-friendly version

Fig. 1 (on pg. 13): the second mode of lead width for Arctic winter by beam \#3 is offset from those of beam \#1 and \#5 by 10 meters [top-right and bottom right sub-figure of subfigure (a)] . Why? 


\section{References:}

Kwok, R., Kacimi, S., Markus, T., Kurtz, N. T., Studinger, M., Sonntag, J. G., et al. (2019). ICESatâĂŘ2 surface height and sea ice freeboard assessed with ATM lidar acquisitions from Operation IceBridge. Geophysical Research Letters, 46, 11,22811,236. https://doi.org/10.1029/2019GL084976

Petty, A. A., Kurtz, N. T., Kwok, R., Markus, T., \& Neumann, T. A. (2020). Winter Arctic sea ice thickness from ICESatâĂŘ2 freeboards. Journal of Geophysical Research: Oceans, 125, e2019JC015764. https://doi.org/10.1029/2019JC015764

Interactive comment on The Cryosphere Discuss., https://doi.org/10.5194/tc-2020-174, 2020. 